\title{
CLOSED PLANAR CURVES WITHOUT INFLECTIONS
}

\author{
SHUNTARO OHNO, TETSUYA OZAWA, AND MASAAKI UMEHARA
}

(Communicated by Daniel Ruberman)

\begin{abstract}
We define a computable topological invariant $\mu(\gamma)$ for generic closed planar regular curves $\gamma$, which gives an effective lower bound for the number of inflection points on a given generic closed planar curve. Using it, we classify the topological types of locally convex curves (i.e. closed planar regular curves without inflections) whose numbers of crossings are less than or equal to five. Moreover, we discuss the relationship between the number of double tangents and the invariant $\mu(\gamma)$ of a given $\gamma$.
\end{abstract}

\section{INTRODUCTION}

In this paper, curves are always assumed to be regular (i.e. immersed). The well-known Fabricius-Bjerre [3] theorem asserts (see also [5]) that

$$
d_{1}(\gamma)-d_{2}(\gamma)=\#_{\gamma}+\frac{i_{\gamma}}{2}
$$

holds for closed curves $\gamma$ satisfying suitable genericity assumptions, where $d_{1}(\gamma)$ (resp. $d_{2}(\gamma)$ ) is the number of double tangents of the same side (resp. opposite sides) and $\#_{\gamma}$ and $i_{\gamma}$ are the number of crossings and the number of inflections on $\gamma$, respectively. See Figure 1 .

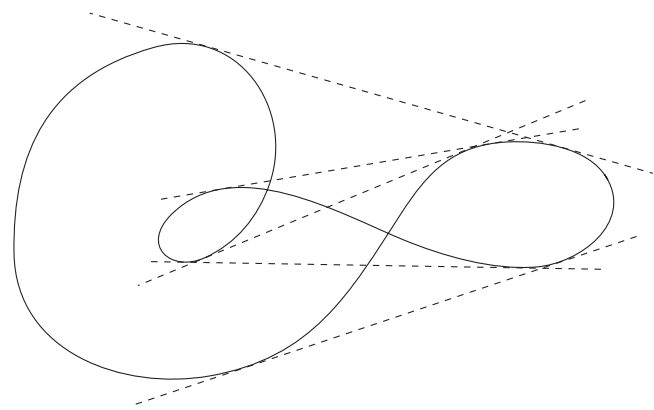

Figure 1. A curve with $\#_{\gamma}=2, i_{\gamma}=2, d_{1}=4$ and $d_{2}=1$.

However, for arbitrarily given nonnegative integers $d_{1}, d_{2}, n$ and $i$ satisfying $d_{1}-$ $d_{2}=n+i / 2$, there might not exist a corresponding curve, in general. As an

Received by the editors December 21, 2010 and, in revised form, June 21, 2011 and June 26, 2011.

2010 Mathematics Subject Classification. Primary 53A04; Secondary 53A15, 53C42.

Key words and phrases. Plane curve, inflection point, double tangent.

The third author was partially supported by the Grant-in-Aid for Scientific Research (A) No. 22244006, Japan Society for the Promotion of Science. 
affirmative answer to the Halpern conjecture in [6], the second author [8] proved the inequality

$$
d_{1}(\gamma)+d_{2}(\gamma) \leq \#_{\gamma}\left(2 \#_{\gamma}-1\right)
$$

for closed curves without inflections (see also Remark 3.4). It is then natural to expect that there might be further obstructions for the topology of closed planar curves without inflections. In this paper, we define a computable topological invariant $\mu(\gamma)$ for closed planar curves. By applying the Gauss-Bonnet formula, we show the inequality $i_{\gamma} \geq \mu(\gamma)$, which is sharp at least for closed curves satisfying $\#_{\gamma} \leq 4$. In fact, for such a $\gamma$, there exists a closed curve $\sigma$ which has the same topological type as $\gamma$ such that $i_{\sigma}=\mu(\sigma)$. As an application, we classify the topological types of closed planar curves satisfying $i_{\gamma}=0$ and $\#_{\gamma} \leq 5$. Moreover, we also introduce an invariant $I(\gamma)$ of a curve $\gamma$ and discuss its relation to the number of double tangents of $\gamma$.

\section{Preliminaries AND main Results}

We denote by $\boldsymbol{R}^{2}$ the Euclidean plane, and by $S^{2}$ the unit sphere in $\boldsymbol{R}^{3}$. A closed curve $\gamma$ in $\boldsymbol{R}^{2}$ or $S^{2}$ is called generic if

(1) all crossings are transversal self-intersection points of multiplicity two, and

(2) the zeroes of curvature are nondegenerate.

By stereographic projection, we identify $S^{2}=\boldsymbol{R}^{2} \cup\{\infty\}$. Two generic closed curves $\gamma_{1}$ and $\gamma_{2}$ in $\boldsymbol{R}^{2}$ (in $S^{2}$ ) are called geotopic, or said to have the same topological type, in $\boldsymbol{R}^{2}$ (resp. in $S^{2}$ ) if there is an orientation-preserving auto diffeomorphism $\varphi$ of $\boldsymbol{R}^{2}$ (resp. on $S^{2}$ ) such that $\operatorname{Im} \gamma_{2}=\varphi\left(\operatorname{Im} \gamma_{1}\right)$. This induces an equivalence relation on the set of closed curves. We denote equivalence classes by $\left\langle\gamma_{1}\right\rangle$ (resp. $\left.\left[\gamma_{1}\right]\right)$. We fix a closed regular curve $\gamma: S^{1} \rightarrow \boldsymbol{R}^{2}$. A point $c \in S^{1}$ is called an inflection point of $\gamma$ if $\operatorname{det}(\dot{\gamma}(t), \ddot{\gamma}(t))$ vanishes at $t=c$. We denote by $i_{\gamma}$ the number of inflection points on $\gamma$. A closed curve $\gamma:[0,1] \rightarrow \boldsymbol{R}^{2}$ is called locally convex if $i_{\gamma}=0$. Whitney [12] proved that any two closed curves are regularly homotopic if and only if their rotation indices coincide. So then one can ask if this regular homotopy can be chosen to preserve local convexity when the given two curves are both locally convex. In fact, one can easily prove this by a modification of Whitney's argument, which has been pointed out in [9, page 35, Exercise 11]. For the sake of the reader's convenience we shall outline the proof:

Proposition 1.1. Let $\gamma_{1}$ and $\gamma_{2}$ be two locally convex closed regular curves. Suppose that $\gamma_{1}$ has the same rotation index as $\gamma_{2}$. Then there exists a continuous family of closed curves $\left\{\Gamma_{\varepsilon}\right\}_{\varepsilon \in[0,1]}$ such that

(1) $\Gamma_{0}=\gamma_{1}$ and $\Gamma_{1}=\gamma_{2}$,

(2) each $\Gamma_{\varepsilon}(\varepsilon \in[0,1])$ is a locally convex closed regular curve.

Proof. Suppose that the curves $\gamma_{j}(j=1,2)$ are both positively curved and have the same rotation indices. We change, if necessary, the parametrizations of $\gamma_{j}$ so that the tangent vectors $\dot{\gamma}_{j}(t)$ are positive scalar multiples of $\left(\begin{array}{c}\cos t \\ \sin t\end{array}\right)$. Define a homotopy $\Gamma_{\varepsilon}$ between $\gamma_{1}$ and $\gamma_{2}$ by $\Gamma_{\varepsilon}(t):=(1-\varepsilon) \gamma_{1}(t)+\varepsilon \gamma_{2}(t)$. It is easy to verify that $\left\{\Gamma_{\varepsilon}\right\}_{\varepsilon \in[0,1]}$ satisfies the required conditions (1) and (2). 
For a given generic closed curve $\gamma$, we set

$$
I(\gamma):=\min _{\sigma \in\langle\gamma\rangle} i_{\sigma}
$$

Since $i_{\gamma}$ is an even number, so is $I(\gamma)$. Inflection points on curves in $\boldsymbol{R}^{2}$ correspond to singular points on their Gauss maps. So it is natural to ask about the existence of topological restrictions on closed curves without inflections; in other words, we are interested in the topological type of closed curves $\gamma$ satisfying $I(\gamma)=0$.

There are explicit combinatorial procedures for determining generic closed spherical curves with a given number of crossings, as in Carter [4] and Cairns and Elton [2] (see also Arnold [1]). We denote by $\#_{\gamma}$ the number of crossings for a given generic closed curve. In this paper, we use the table of closed spherical curves with $\#_{\gamma} \leq 5$ given in the appendix of [7].

For example, the table of closed spherical curves with $\#_{\gamma} \leq 2$ is given in Figure2, where $1_{2}$ (resp. $2_{2}$ ) means that the corresponding curve has 2-crossings and appears in the table of curves in [7] with $\#_{\gamma}=2$ primary (resp. secondary).

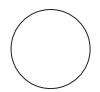

[10]

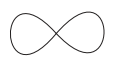

$\left[1_{1}\right]$

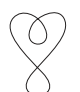

$\left[1_{2}\right]$

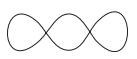

$\left[22_{2}\right]$

FiguRE 2. Spherical closed curves with $\#_{\gamma} \leq 2$.

Moving the position of $\infty$ via motions in $S^{2}=\boldsymbol{R}^{2} \cup\{\infty\}$, we get the table of closed planar curves with $\#_{\gamma} \leq 2$ as in Figure 3 . For example $1_{1}$ and $1_{1}^{b}$ (resp. $2_{2}$, $2_{2}^{b}$ and $2_{2}^{c}$ ) are equivalent to $\left[1_{1}\right]$ (resp. $\left[2_{2}\right]$ ) as spherical curves. Similarly, using the table of spherical curves with $\#_{\gamma} \leq 5$ given in [7, we prove the following theorem. The authors do not know of any references that give such a classification of generic locally convex curves.
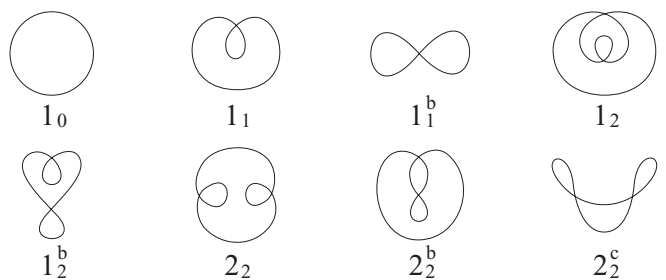

Figure 3. Planar closed curves with $\#_{\gamma} \leq 2$.

Theorem 1.2. For every generic closed regular curve $\gamma$ in $\boldsymbol{R}^{2}$, the inequality

$$
I(\gamma) \geq \mu(\gamma)
$$

holds. (The definition of $\mu(\gamma)$ is given in (2.2).) Moreover, $I(\gamma)=0$ if and only if $\mu(\gamma)=0$ under the assumption that $\#_{\gamma} \leq 5$.

We prove this assertion in Section 2. In particular, the number of equivalence classes of closed locally convex curves with $\#_{\gamma} \leq 5$ is 76 (see Figure 3 and Figures 13, 15 and 16 in Section 3). For example, in Figure 3 the curves of type $1_{0}, 1_{1}, 1_{2}, 2_{2}$ satisfy $I(\gamma)=0$, and the remaining $1_{1}^{b}, 1_{2}^{b}, 2_{2}^{b}, 2_{2}^{c}$ satisfy $I(\gamma)=2$. In 
Figure 13, the curve of type $6_{3}^{a}$ is of the same topological type as $6_{3}^{b}$ as a spherical curve, which is obtained from the 6 th curve in the table of curves with $\#_{\gamma}=2$ in the appendix of [7].

Corollary 1.3. A generic closed curve $\gamma$ with $\#_{\gamma} \leq 4$ satisfies $I(\gamma)=\mu(\gamma) \leq 2$, unless the topological type of $\gamma$ is as in Figure 4 .

The proof of this corollary is given at the end of section 2 .

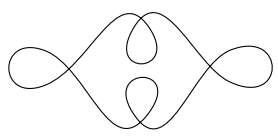

Figure 4. A curve with $\#_{\gamma}=4$ and $I(\gamma)=\mu(\gamma)=4$.

A table of closed curves $\gamma$ for $\#_{\gamma} \leq 3$ is given in Figures 3, 13 and 14 and drawn to be $i_{\gamma}=\mu(\gamma)$.

\section{Definition of the invariant $\mu(\gamma)$}

We fix a generic closed curve $\gamma: S^{1} \rightarrow \boldsymbol{R}^{2}$, where $S^{1}=\boldsymbol{R} / \boldsymbol{Z}$, that is, $\gamma$ is a periodic map satisfying $\gamma(t)=\gamma(t+1)$ for $t \in \boldsymbol{R}$. We set $\#_{\gamma}=m$. We may suppose that $\gamma(0)=\gamma(1)$ is one of the crossings of $\gamma$. Let

$$
0=c_{1} \prec \cdots \prec c_{2 m}\left(\prec c_{1}\right)
$$

be the inverse image of the crossings of $\gamma$, which consists of $2 m$ points in $S^{1}=\boldsymbol{R} / \boldsymbol{Z}$. Here, $\prec$ denotes the cyclic order of $S^{1}$. We set

$$
S_{\gamma}^{1}:=S^{1} \backslash\left\{c_{1}, \ldots, c_{2 m}\right\} .
$$

To introduce the invariant $\mu(\gamma)$, we define special subsets on the curves called ' $n$-gons':

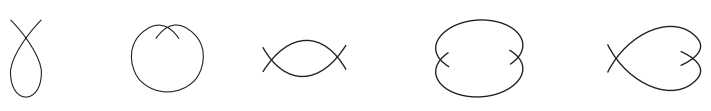

FiguRE 5. Shells and leaves.

Definition 2.1. Let $n(\geq 3)$ be an integer. A disjoint union of $n$ proper closed intervals

$$
J:=\left[a_{1}, b_{1}\right] \cup \cdots \cup\left[a_{n}, b_{n}\right]
$$

on $S^{1}$ is called an $n$-gon if $a_{1}, b_{1}, \cdots, a_{n}, b_{n} \in\left\{c_{1}, \ldots, c_{2 m}\right\}$ and the image $\gamma(J)$ is a piecewise smooth simple closed curve in $\boldsymbol{R}^{2}$. The simply connected domain bounded by $\gamma(J)$ is called the interior domain of the $n$-gon. An $n$-gon is called admissible if at most two of the $n$ interior angles of $D$ are less than $\pi$.

We denote by $\mathcal{G}_{n}(\gamma)$ the set of all admissible $n$-gons, and set

$$
\mathcal{G}(\gamma):=\bigcup_{n=1}^{\infty} \mathcal{G}_{n}(\gamma)
$$

Each element of $\mathcal{G}(\gamma)$ is called an admissible polygon. A 1-gon is called a shell (cf. Figure 5). A 2-gon is called a leaf and a 3-gon is called a triangle (cf. Figure 66). 

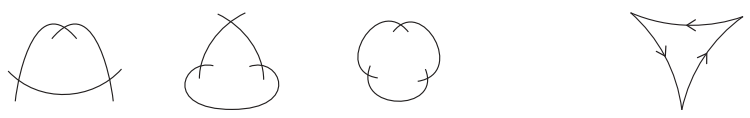

Figure 6. Admissible triangles and a nonadmissible triangle.

All shells and all leaves are admissible. However, a triangle whose interior angles are all acute is not admissible.

We fix an admissible $n$-gon $J:=\left[a_{1}, b_{1}\right] \cup \cdots \cup\left[a_{n}, b_{n}\right]$. Then $\gamma(J)$ is a piecewise smooth simple closed curve in $\boldsymbol{R}^{2}$. We give an orientation of $\gamma(J)$ so that the interior domain of $\gamma(J)$ is on the left-hand side of $\gamma(J)$. This orientation induces an orientation on $\left[a_{i}, b_{i}\right]$ for each $i=1, \ldots, n$. We call $\left[a_{i}, b_{i}\right]$ a positive interval (resp. negative interval) if the orientation of the interval $\left[a_{i}, b_{i}\right]$ coincides with (resp. does not coincide with) the orientation of $\left[a_{i}, b_{i}\right]$ induced from $\gamma$.

Let $t$ be a point on $J \backslash\left\{a_{1}, b_{1}, \ldots, a_{n}, b_{n}\right\}=\bigcup_{i=1}^{n}\left(a_{i}, b_{i}\right)$. Then $t$ belongs to an open interval $\left(a_{i}, b_{i}\right)$ for some $i=1, \ldots, n$. Then we set

$$
\varepsilon_{J}(t):= \begin{cases}1 & \text { if }\left[a_{i}, b_{i}\right] \text { is positive } \\ -1 & \text { if }\left[a_{i}, b_{i}\right] \text { is negative. }\end{cases}
$$

Definition 2.2. An admissible $n$-gon is called positive (resp. negative) if $\varepsilon_{J}(t)>0$ (resp. $\varepsilon_{J}(t)<0$ ) holds for each $t \in J \backslash\left\{a_{1}, b_{1}, \ldots, a_{n}, b_{n}\right\}$.

The notions of positivity and negativity of shells that were used in [1] and [7] are not the same as ours. The following assertion is the key to proving the inequality (1.2):

Lemma 2.3. Let $J:=\left[a_{1}, b_{1}\right] \cup \cdots \cup\left[a_{n}, b_{n}\right]$ be an admissible $n$-gon. Then there exists an interval $\left[a_{i}, b_{i}\right](1 \leq i \leq n)$ and a point $c \in\left(a_{i}, b_{i}\right)$ such that $\operatorname{sgn}\left(\kappa_{\gamma}(c)\right)=\varepsilon_{J}(c)$, where $\kappa_{\gamma}(t):=\operatorname{det}(\dot{\gamma}(t), \ddot{\gamma}(t)) /|\dot{\gamma}|^{3}$ is the curvature of $\gamma$ and $\operatorname{sgn}\left(\kappa_{\gamma}(c)\right)$ is the sign of the real number $\kappa_{\gamma}(c)$.

Proof. Let $A_{1}, A_{2}, \cdots, A_{n}$ be the interior angles of the interior domain of $J$, and set $\Gamma=\gamma(J)$, which we regard as an oriented piecewise smooth simple closed curve with counterclockwise orientation. We first consider the case $n \geq 3$. We take $s$ to be the arclength parameter of $\Gamma$. Let $s=s_{1}, \ldots, s_{n}$ be the points where $d \Gamma / d s$ is discontinuous. We denote by $\kappa_{\Gamma}(s)\left(s \neq s_{1}, \ldots, s_{n}\right)$ the curvature of the curve $\Gamma$. Then, the Gauss-Bonnet formula yields that

$$
\int_{J} \kappa_{\Gamma}(s) d s=-(n-2) \pi+\sum_{i=1}^{n} A_{i} .
$$

Since $J$ is admissible, we may assume that $A_{1}, \ldots, A_{n-2} \geq \pi$, and so $\int_{J} \kappa_{\Gamma}(s) d s>0$. Then there exist an index $i(1 \leq i \leq n)$ and $c \in\left[a_{i}, b_{i}\right]$ such that $\kappa_{\Gamma}(c)>0$. We denote by $\kappa_{\gamma}(s)$ the Gaussian curvature of $\gamma$ at $\Gamma(s)$. Then we have that $\varepsilon_{J}(c) \kappa_{\gamma}(c)=\kappa_{\Gamma}(c)>0$, which proves the assertion. If $n=1$ (resp. $\left.n=2\right)$, then replacing $A_{1}, A_{2}$ by $A_{2}, A_{3}$ (resp. $A_{1}$ by $A_{3}$ ), we may consider $\Gamma$ as a triangle of $A_{1}=A_{2}=\pi$ (resp. $\left.A_{1}=\pi\right)$. Then the same proof can be applied.

Remark 2.4. As seen in Figure 6, right, the nonadmissible triangle (i.e. triangle with sum of three angles less than $\pi$ ) can be realized only by negative curvature.

We now define the invariant $\mu(\gamma)$ mentioned in the introduction: If $\gamma$ is a simple closed curve, we set $\mu(\gamma):=0$. We assume that $\gamma$ has at least one crossing. 
Definition 2.5. A function $\Phi: S_{\gamma}^{1} \rightarrow\{0,-1,1\}$ is called an admissible function with respect to $\gamma$ if it satisfies the following conditions:

(1) $\operatorname{supp}(\Phi):=\Phi^{-1}(1) \cup \Phi^{-1}(-1)$ is a finite set, and

(2) for each $J \in \mathcal{G}(\gamma)$, there exists $t \in \operatorname{supp}(\Phi)$ such that $t \in J$ and $\varepsilon_{J}(t)=$ $\Phi(t)$.

A point $t \in \operatorname{supp}(\Phi)$ is called a positive point (resp. negative point) if $\Phi(t)>0$ (resp. $\Phi(t)<0$ ). We denote by $\mathcal{A}$ the set of all admissible functions of $\gamma$.

We fix an admissible function $\Phi \in \mathcal{A}$. Then we have an $\operatorname{expression} \operatorname{supp}(\Phi)=$ $\left\{u_{1}, \ldots, u_{\ell}\right\}$ such that

$$
u_{1} \prec u_{2} \prec \cdots \prec u_{\ell}\left(\prec u_{1}\right) .
$$

Let $\mu(\Phi)$ be the number of sign changes of the cyclic sequence

$$
\Phi\left(u_{1}\right), \Phi\left(u_{2}\right), \cdots, \Phi\left(u_{\ell}\right), \Phi\left(u_{1}\right),
$$

which is a positive even integer. Then we set

$$
\mu(\gamma):=\min _{\Phi \in \mathcal{A}} \mu(\Phi)
$$

Proof of the inequality (1.2). Let $\gamma$ be a generic closed curve in $\boldsymbol{R}^{2}$. We take a curve $\sigma \in\langle\gamma\rangle$ such that $i_{\sigma}=I(\gamma)$. Without loss of generality, we may assume that $i_{\gamma}=$ $I(\gamma)$. We can take a point $c_{J} \in[0,1]$ for each $J \in \mathcal{G}$ so that $\operatorname{sgn}\left(\kappa_{\gamma}\left(c_{J}\right)\right)=\varepsilon_{J}\left(c_{J}\right)$, and that $c_{J} \neq c_{K}$ if $J \neq K(K \in \mathcal{G})$. We define a function $\Phi: S_{\gamma}^{1} \rightarrow\{0,-1,1\}$ by

$$
\Phi(t):= \begin{cases}\operatorname{sign}\left(\kappa_{\gamma}\left(c_{J}\right)\right) & \text { if } t=c_{J} \text { for some } J \in \mathcal{G}(\gamma), \\ 0 & \text { otherwise. }\end{cases}
$$

Then, $\Phi$ is an admissible function. Since $i_{\gamma}=I(\gamma)$, the curvature function of $\gamma$ changes sign at most $I(\gamma)$ times. So we have that $\mu(\Phi) \leq I_{\gamma}$; in particular, $\mu(\gamma) \leq I_{\gamma}$.

Also we have the following assertion:

Proposition 2.6. Let $\gamma$ be a generic closed curve in $\boldsymbol{R}^{2}$. Then $\mu(\gamma)$ is a nonnegative even integer, as is $I(\gamma)$. Moreover, $\mu(\gamma)>0$ holds if and only if $\mathcal{G}(\gamma)$ contains a positive polygon and a negative polygon at the same time.

Proof. Since the number of sign changes of a cyclic sequence of real numbers is always even, $\mu(\gamma)$ is also even. Moreover, if $\gamma$ has a positive (resp. negative) polygon, each admissible function $\Phi$ must take a positive (resp. negative) value. So the existence of two distinct polygons of opposite sign implies that $\mu(\gamma) \geq 2$.

Now, we prove the converse. A closed curve which is not a simple closed curve $1_{0}$ has at least one shell, and a shell is necessarily a positive or a negative polygon. Suppose that $\gamma$ has no negative polygons. Then we can choose a point $c_{J} \in J \cap S_{\gamma}^{1}$ for each admissible polygon $J \in \mathcal{G}(\gamma)$ such that $\varepsilon_{J}\left(c_{J}\right)>0$. If we set

$$
\Phi(t):= \begin{cases}\varepsilon_{J}\left(c_{J}\right) & \text { if } t=c_{J} \text { for some } J \in \mathcal{G}(\gamma), \\ 0 & \text { otherwise, }\end{cases}
$$

then $\Phi$ is an admissible function, and $\mu(\Phi)=0$. Thus we have $\mu(\gamma)=0$. This proves the converse. 
To show the computability of the invariant $\mu(\gamma)$, we fix $4 m\left(m=\#_{\gamma}\right)$ points $t_{i}, s_{i}(i=1, \ldots, 2 m)$ satisfying

$$
c_{1} \prec t_{1} \prec s_{1} \prec c_{2} \prec \cdots \prec c_{2 m} \prec t_{2 m} \prec s_{2 m}\left(\prec c_{1}\right) .
$$

The following lemma holds:

Lemma 2.7. For each admissible function $\Phi$ of $\gamma$, there exists an admissible function $\Psi$ satisfying the following properties:

(1) $\operatorname{supp}(\Psi) \subset\left\{t_{1}, s_{1}, \ldots, t_{2 m}, s_{2 m}\right\}$,

(2) $\mu(\Psi) \leq \mu(\Phi)$.

Proof. We fix an interval $U=\left(c_{i}, c_{i+1}\right)$, where $c_{2 m+1}:=c_{1}$. If $\Phi$ takes only nonnegative (resp. nonpositive) values on $U$, then we set

$$
\Psi(t):= \begin{cases}1(\text { resp. }-1) & \text { if } t=t_{i}, \\ 0 & \text { if } t \in U \backslash\left\{t_{i}\right\} .\end{cases}
$$

If $\operatorname{supp}(\Phi) \cap U$ contains two points $v_{1}, v_{2}$ such that $v_{1}<v_{2}$ and $\Phi\left(v_{1}\right)=-\Phi\left(v_{2}\right)$, then we set

$$
\Psi(t):= \begin{cases}\Phi\left(v_{1}\right) & \text { if } t=t_{i} \\ \Phi\left(v_{2}\right) & \text { if } t=s_{i} \\ 0 & \text { otherwise. }\end{cases}
$$

Since $U$ is arbitrary, we get a function $\Psi$ defined on $S_{\gamma}^{1}$. Since $U \cap J$ coincides with either $U$ or an empty set for each $J \in \mathcal{G}(\gamma), \Psi$ is an admissible function, and one can easily verify $\mu(\Psi) \leq \mu(\Phi)$.

Remark 2.8 (Computability of the invariant $\mu(\gamma)$ ). The function $\Psi$ obtained in Lemma 2.7 is called a reduction of $\Phi$. ( $\Psi$ may not be uniquely determined from $\Phi$, since $\operatorname{supp}(\Phi) \cap U$ might consist of more than two points.) By definition, there exists an admissible function $\Phi$ such that $\mu(\Phi)=\mu(\gamma)$. By Lemma 2.7, there is a reduction of such a function $\Phi$. Thus the invariant $\mu(\gamma)$ is attained by a reduced function $\Psi$. Since the number of reduced admissible functions is at most $3^{4 m}$, the invariant $\mu(\gamma)$ can be computed in a finite number of steps.

Remark 2.9 (A flexibility of the reduced admissible function). In the above construction of the function $\Psi$ via $\Phi$ we may set

$$
\Psi(t):= \begin{cases}\Phi\left(v_{2}\right) & \text { if } t=t_{i}, \\ \Phi\left(v_{1}\right) & \text { if } t=s_{i}, \\ 0 & \text { otherwise }\end{cases}
$$

when $\operatorname{supp}(\Phi) \cap U$ contains two points $v_{1}, v_{2}$ such that $v_{1}<v_{2}$ and $\Phi\left(v_{1}\right)=-\Phi\left(v_{2}\right)$. Then $\Psi$ is also an admissible function. This modification of $\Psi$ can be done for each fixed interval $U=\left(c_{i}, c_{i+1}\right)$. However, after the operation, it might not hold that $\mu(\Psi) \leq \mu(\Phi)$.

Proposition 2.10. Let $\gamma$ be a generic closed curve in $\boldsymbol{R}^{2}$. Then it satisfies

$$
\mu(\gamma) \leq 2 \# \gamma .
$$

Proof. As pointed out in Remark 2.8, there exists a reduced admissible function $\Psi$ such that $\mu(\Psi)=\mu(\gamma)$. Since $\mu(\Psi) \leq 4 m$, we get the estimate $\mu(\gamma) \leq 4 \#_{\gamma}$. However, we can improve it as follows: As pointed out in Remark 2.9. one can 
replace the values $\Psi\left(t_{i}\right), \Psi\left(s_{i}\right)$ by $-\Psi\left(t_{i}\right),-\Psi\left(s_{i}\right)$ whenever $\Psi\left(t_{i}\right)=-\Psi\left(s_{i}\right)$ for each $i=1, \ldots, 2 m$. So using this modification inductively for $i=1, \ldots, 2 m$ if necessary, we can modify $\Psi$ so that $\mu(\Psi) \leq 2 m$, and then we have $\mu(\gamma) \leq 2 \#(\gamma)$.

Remark 2.11. If $\gamma$ is a lemniscate $1_{1}^{b}$, then $\mu(\gamma)=2 \#_{\gamma}=2$ holds. However, the authors do not know of any other example satisfying the equality $\mu(\gamma)=2 \#_{\gamma}$ (cf. Question 3).

Example 2.12 (Curves with a small number of intersections). Here, we demonstrate how to determine $\mu(\gamma)$ when $\#_{\gamma} \leq 2$. In the eight classes of curves in Figure 3, four classes have been drawn without inflections. So $I(\gamma)=0$ holds for these four curves. Each of the remaining four curves satisfies $\mu(\gamma)>0$, by Proposition 2.6. On the other hand, these four curves in Figure 3 have been drawn with exactly two inflections. Thus we can conclude that they satisfy $\mu(\gamma)=2$. Now, let $\gamma$ be a curve as in Figure 4. Then $\gamma$ has four disjoint shells, two of which are positive, and the other two are negative. So we can conclude that $\mu(\gamma) \geq 4$. Since $\gamma$ as in Figure 4 has exactly four inflections, we can conclude that $I(\gamma)=\mu(\gamma)=4$.
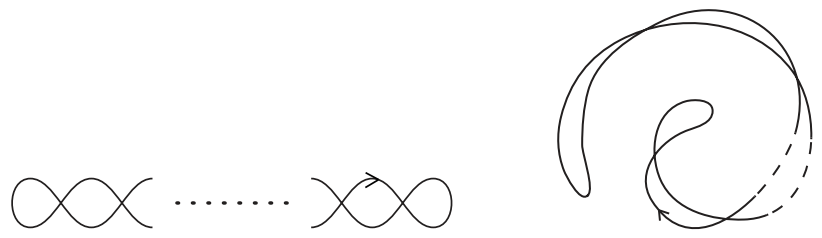

FIGURE 7. A chain-like curve and its realization with $i_{\gamma}=2$.

Example 2.13 (Chain-like curves). We consider a curve $\gamma$ with $\#_{\gamma}=n(n \geq 1)$ as in Figure 7, left. This curve has two shells and $n-1$ leaves, including a positive shell and a negative leaf, which are disjoint. Thus $I(\gamma) \geq \mu(\gamma) \geq 2$ holds. As in Figure 7, right, this curve can be drawn along a spiral with two inflections. So we can conclude that $I(\gamma)=\mu(\gamma)=2$. In this manner, drawing curves along a spiral is often useful to reduce the number of inflection points. Several useful techniques for drawing curves with a restricted number of inflections are mentioned in Halpern [6. Section 4].

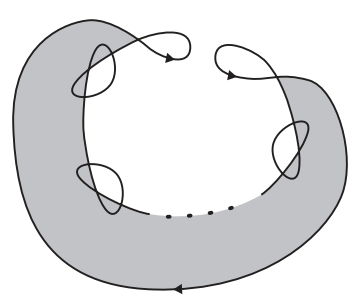

Figure 8. A figure of the curve $\gamma_{n}$.

Example 2.14 (Curves with a negative $n$-gon). We consider a curve $\gamma_{n}$ as in Figure 8, which has several positive polygons, but only one negative admissible $n$ gon, which is marked in gray in Figure 8. So we can conclude that $I(\gamma)=\mu(\gamma)=2$, 
as in Figure 8. This example shows that an $n$-gon $(n \geq 4)$ is needed to find a curve that cannot be locally convex.

The admissibility of a polygon in the curve is important for the definition of the invariant $\mu$. The curve as in Figure 9, left, has negative polygons which are not admissible, and it can be realized without inflections as in Figure 9, right.
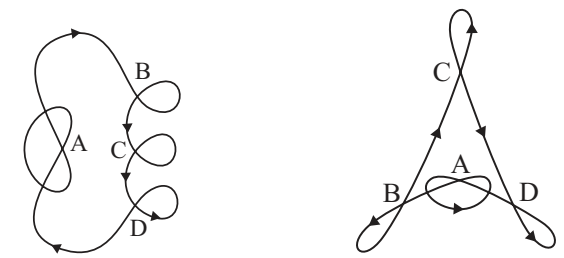

Figure 9. A curve satisfying $i_{\gamma}=0$.

Example 2.15 (A curve with an effective leaf which is neither positive nor negative). We consider a curve $\gamma$ as in Figure 10, This curve has 5 crossings and exactly two positive shells at $A$ and $B$. It also has a negative shell at $C$. Thus $\mu \geq 2$ by Proposition 2.6. We show that $\mu(\gamma)=4$ by way of a contradiction: There exist two positive points $p_{1}$ and $p_{2}$ on the two positive shells at $A$ and $B$, respectively. There is a unique simple arc $\Gamma$ bounded by $A$ and $B$ which passes through $D$ and $E$. Suppose that $\mu(\gamma)=2$. Then there are no negative points on $\Gamma$. Now we look at the negative leaf with vertices $A$ and $D$. In Figure 10, this leaf is marked in gray. Since $\Gamma$ does not contain a negative point, there must be a negative point $m_{1}$ between $A$ and $D$ on this leaf. Since the curve has a symmetry, applying the same argument to the negative leaf at $B$ and $E$, there is another negative point $m_{2}$ between $E$ and $C$.

Finally, we look at a leaf with vertices $D$ and $E$, which is neither positive nor negative. Since $\Gamma$ has no negative point, it must have a positive point $p_{3}$ on the arc on the right-hand side of the leaf. Since the sequence $p_{1}, m_{1}, p_{3}, m_{2}, p_{2}$ changes sign four times, this gives a contradiction. Thus $\mu(\gamma) \geq 4$. Since the curve can be drawn with exactly four inflections as in Figure 10, we can conclude that $I(\gamma)=\mu(\gamma)=4$. In this example, an admissible polygon which is neither positive nor negative plays a crucial role to estimate the invariant $I(\gamma)$ by using $\mu(\gamma)$.

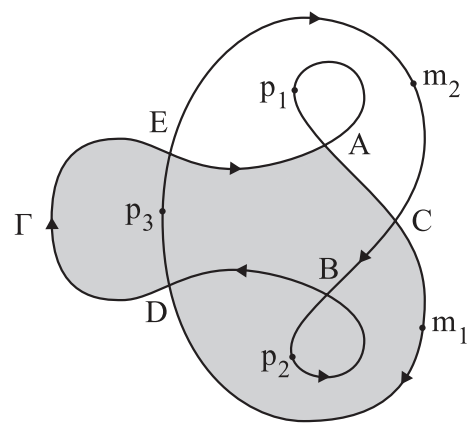

FiguRE 10. A curve satisfying $i_{\gamma}=4$ and $\#_{\gamma}=5$. 
Proof of the second assertion of Theorem 1.2. The table of spherical curves up to $\#_{\gamma} \leq 5$ is given in the appendix of [7]. By moving the position of $\infty$ in $S^{2}=$ $\boldsymbol{R}^{2} \cup\{\infty\}$, we get the table of planar curves up to $\#_{\gamma} \leq 5$ and can compute the invariant $\mu(\gamma)$. So we can list the curves with $\mu(\gamma)=0$. By Proposition [2.6, it is sufficient to check for the existence of positive polygons and negative polygons. When $\#_{\gamma} \leq 2$, the number of topological types of such curves is 3 . If $\#_{\gamma}=3,4,5$, then the number of topological types of such curves is $6,16,50$, respectively. After that we can draw the pictures of the curves by hand. If we are able to draw 76 figures of the curves without inflections, the proof is finished. This was accomplished in Figures 13, 15 and 16.

Proof of Corollary 1.3. For curves $\gamma$ with $\mu(\gamma)=0$, we can show that $I(\gamma)=0$ by drawing curves without inflections. On the other hand, when $\mu(\gamma)>0$, we can show that $I(\gamma)=2$ by drawing curves with exactly two inflections, except for the curve $\gamma_{0}$ as in Figure 4. In fact, the curve $\gamma_{0}$ satisfies $I\left(\gamma_{0}\right)=\mu\left(\gamma_{0}\right)=4$ as shown in Example 2.12.

\section{Double tangents And geotopical tightness}

In this section, we would like to give an application.

Definition 3.1. Let $\gamma$ be a generic planar curve. We set

$$
d(\gamma):=d_{1}(\gamma)+d_{2}(\gamma)
$$

Then we define

$$
\delta(\gamma):=\min _{\sigma \in\langle\gamma\rangle} d(\sigma)
$$

which gives the minimum number of double tangents of the curves in the equivalence class $\langle\gamma\rangle$. (As in Figure 11, $d(\sigma)$ might be different from $d(\gamma)$ even if $\sigma \in\langle\gamma\rangle$ and $i_{\gamma}=i_{\sigma}=I(\gamma)$.) A curve $\sigma \in\langle\gamma\rangle$ satisfying $d(\gamma)=\delta(\gamma)$ is called geotopically tight or $g$-tight. We call the integer $\delta(\gamma)$ the $g$-tightness number.
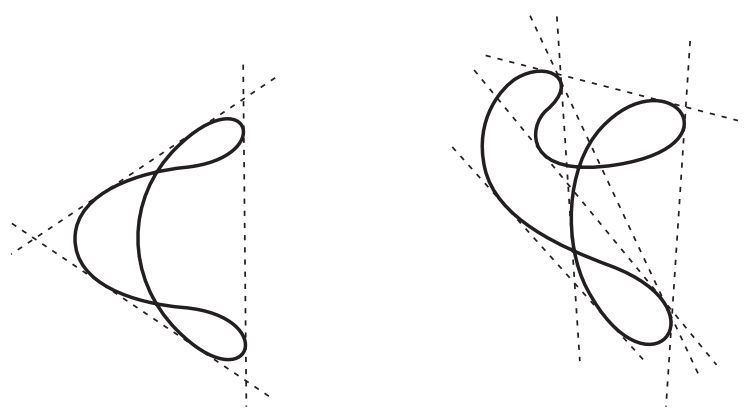

Figure 11. Curves of $2_{2}^{c}$ with $i_{\gamma}=2$ but with different $d$.

The following assertion holds:

Proposition 3.2. It holds that $\delta(\gamma) \geq \#_{\gamma}+I(\gamma) / 2$. 
Proof. By (0.1), we get that

$$
d(\sigma)=d_{1}(\sigma)+d_{2}(\sigma) \geq d_{1}(\sigma)-d_{2}(\sigma)=\#_{\sigma}+\frac{I(\sigma)}{2}
$$

holds for $\sigma \in\langle\gamma\rangle$. Taking the infimum, we get the assertion.

We expect that all locally convex generic closed curves are $g$-tight (see Question 4). Relating this, we can prove the following assertion:

Let $\gamma: \boldsymbol{R} \rightarrow \boldsymbol{R}^{2}$ be a periodic parametrization such that $\gamma(t+1)=\gamma(t)$ holds for $t \in \boldsymbol{R}$. Without loss of generality, we may assume that the curvature of $\gamma$ is positive. Let

$$
x=\gamma\left(s_{1}\right)=\gamma\left(s_{2}\right) \quad\left(0<\left|s_{1}-s_{2}\right|<1\right)
$$

be a crossing of $\gamma$. Replacing $s_{1}$ by $s_{1}-1$ if necessary, we may assume that $s_{1}<s_{2}$ and $\dot{\gamma}\left(s_{2}\right)$ is a positively rotated vector of $\dot{\gamma}\left(s_{1}\right)$ through an angle $\alpha$ with $0<\alpha<\pi$. When the parameter $t$ varies in the interval $\left[s_{1}, s_{2}\right]$, the tangent vector $\dot{\gamma}(t)$ rotates through an angle $2 \pi n_{1}(x)+\alpha$, where $n_{1}(x)$ is a positive integer. Similarly, for the interval $\left[s_{2}, s_{1}+1\right]$, there exists a positive integer $n_{2}(x)$ such that the rotation angle of $\dot{\gamma}(t)$ is equal to $2 \pi n_{2}(x)-\alpha$. The sum $n_{1}(x)+n_{2}(x)$ is the total rotation index of $\gamma$. Denote by $W(x)$ the difference $n_{1}(x)-n_{2}(x)$. We easily recognize that the sum of $W(x)$ for each crossing $x$ of $\gamma$ is a geotopy invariant. The following theorem holds:

Theorem 3.3. The number of double tangents for any locally convex generic closed curve $\gamma$ depends only on its geotopy type. More precisely, the following identity holds:

$$
d_{2}(\gamma)=\sum W(x)
$$

where the sum runs over all crossings $x$ of $\gamma$.

Proof. We give here only an outline of the proof. By a suitable homothetic transformation in $\boldsymbol{R}^{2}$ and a change of the parameter $t$, we may assume that $t$ is the arclength parameter. As in [8], we set $A:=\left\{(t, s) \in S^{1} \times S^{1}: \gamma(t)=\gamma(s)\right.$, and $\left.t \neq s\right\}$. In [8], an integer $\operatorname{Res}(p)$ is defined for each $p=(t, s) \in A$, and is equal to

$$
\operatorname{Res}(p)= \begin{cases}1 & (\text { if } \operatorname{det}(\dot{\gamma}(t), \dot{\gamma}(s))<0) \\ -1 & (\text { if } \operatorname{det}(\dot{\gamma}(t), \dot{\gamma}(s))>0)\end{cases}
$$

Let $a_{i}(t)\left(i=1, \ldots, r_{\gamma}\right)$ be functions in $t$ satisfying $t<a_{1}(t)<\cdots<a_{r_{\gamma}}(t)<t+1$ such that $-\dot{\gamma}(t)=\dot{\gamma}\left(a_{i}(t)\right)$, where $r_{\gamma}(>0)$ is the rotation index of the curve $\gamma$. Let $\Theta: S^{1}:=\boldsymbol{R} / \boldsymbol{Z} \ni t \mapsto(\cos t, \sin t) \in \boldsymbol{R}^{2}$ be the identification map of $S^{1}$ into the unit circle. Define a function $f_{i}: S^{1} \rightarrow S^{1}$ for each $i=1, \cdots, r_{\gamma}$ by

$$
f_{i}(t):=\Theta^{-1}(\dot{c}(t))-\Theta^{-1}\left(\frac{c\left(a_{i}(t)\right)-c(t)}{\left|c\left(a_{i}(t)\right)-c(t)\right|}\right) \text {. }
$$


By Lemma 1 and Lemma 2 in [8, it follows that

$$
d_{2}(\gamma)=\sum_{j=1}^{r_{\gamma}} \operatorname{deg} f_{i}=\sum_{j=2}^{r_{\gamma}-1} \operatorname{deg} f_{i}
$$

(Here, the notation $I(\gamma)$ (resp. $d$ ) used in [8] coincides with $d_{2}(\gamma)$ (resp. $\left.r_{\gamma}\right)$.) On the other hand, as in [8, (4)], it follows that (see Remark 3.5)

$$
\operatorname{deg} f_{j+1}-\operatorname{deg} f_{j}=\sum_{p \in W_{j}} \operatorname{Res}(p),
$$

where $u_{i}(t)=\left(t, a_{i}(t)\right)\left(i=1, \ldots, r_{\gamma}\right)$ and $W_{j}$ is the set of double points between the images of $u_{j}$ and $u_{j+1}$ in $S^{1} \times S^{1}$. Since $\operatorname{deg} f_{1}=0$, (3.4) and (3.5) yield that

$$
d_{2}=\left(r_{\gamma}-2\right) \operatorname{deg} f_{1}+\sum_{j=2}^{r_{\gamma}-1}\left(r_{\gamma}-j\right)\left(\operatorname{deg} f_{j}-\operatorname{deg} f_{j-1}\right)=\sum_{j=1}^{r_{\gamma}-2}\left(r_{\gamma}-j\right) \sum_{p \in W_{j}} \operatorname{Res}(p) .
$$

Let $k(p)(p \in A)$ be the integer such that $p \in W_{k(p)}$. We denote by $A_{+}(\subset A)$ the set consisting of points $p \in A$ satisfying $\operatorname{Res}(p)=1$. For the sake of simplicity, we set $p^{\prime}=\operatorname{sym}(p)$, where $\operatorname{sym}(t, s):=(s, t)$ (cf. [8, page 557]). Since $\operatorname{Res}\left(p^{\prime}\right)=-1$, we have that

$$
d_{2}=\sum_{p \in A}\left(r_{\gamma}-k(p)\right) \operatorname{Res}(p)=\sum_{p \in A_{+}}\left(k\left(p^{\prime}\right)-k(p)\right) .
$$

One can now obtain the formula (3.3) via the following key observation:

$$
p \in \begin{cases}W_{n_{2}(x)} & \text { if } \operatorname{Res}(p)=1, \\ W_{n_{1}(x)} & \text { if } \operatorname{Res}(p)=-1,\end{cases}
$$

where $p=(t, s) \in A$, and $x=\gamma(t)=\gamma(s)$.

Remark 3.4. The rotation index $r_{\gamma}$ of $\gamma$, which is equal to $n_{1}(x)+n_{2}(x)$ at each crossing $x$, is less than or equal to $\#_{\gamma}+1$ (cf. [12]). Since $n_{2}(x) \geq 1$, the formula (3.3) implies that

$$
\begin{aligned}
d_{2}(\gamma) & =\sum W(x)=\sum\left(n_{1}(x)+n_{2}(x)\right)-2 \sum n_{2}(x)=\#_{\gamma} r_{\gamma}-2 \sum n_{2}(x) \\
& \leq \#_{\gamma} r_{\gamma}-2 \#_{\gamma}=\#_{\gamma}\left(\#_{\gamma}+1\right)-2 \#_{\gamma}=\#_{\gamma}\left(\#_{\gamma}-1\right),
\end{aligned}
$$

which reproves Halpern's conjecture in [6].

Remark 3.5. The signs of the equation [8, (4)] contain misprints. The equation should be read as

$$
\begin{aligned}
\operatorname{Deg}\left(u_{i}\right)-\operatorname{Deg}\left(u_{j}\right) & =\sum_{p \in h\left(S^{1} \times[0,1]\right)} \operatorname{Res}\left(p_{1}\right) \\
& =\text { the number of } p \in h\left(S^{1} \times[0,1]\right) \text { with } \operatorname{Res}\left(p_{1}\right)=1 \\
& \leq D(c) .
\end{aligned}
$$

The other conclusions in 8 are not affected by this change. 
Corollary 3.6. $\delta(\gamma)=1,2,2,3,3$ for $\gamma$ of type $1_{1}, 1_{1}^{b}, 1_{2}, 2_{2}^{b}, 2_{2}^{c}$ as in Figure 3 , respectively.

Proof. By Proposition 3.2, $\delta(\gamma) \geq 1,2,2,3,3$ for $\gamma$ of type $1_{1}, 1_{1}^{b}, 1_{2}, 2_{2}^{b}, 2_{2}^{c}$, respectively. On the other hand, the curves given in Figure 3 attain equality in this inequality.

In Figure 3, there are two remaining curves of type $1_{2}^{b}$ and $2_{2}$, whose $g$-tightness numbers have not been specified by the authors. The curve of type $2_{2}$ given in the left of Figure 12 satisfies $d(\gamma)=4$, and we expect that $\delta(\gamma)=4$. On the other hand, the curve of type $1_{2}^{b}$ as in the right of Figure 12 satisfies $d=7$, but one can realize the curve with $d=5$ as in Figure 1. We expect that it might be $g$-tight. If true, $\delta(\gamma)=5$ holds.
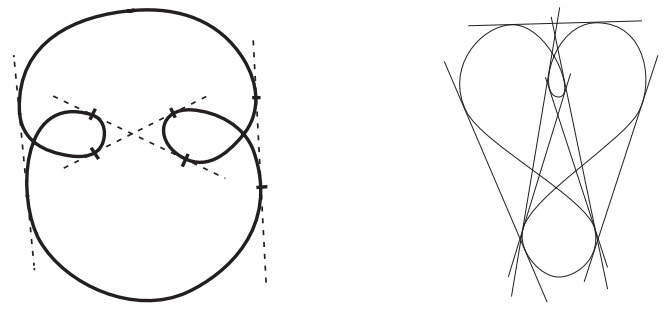

Figure 12. Curves of type $2_{2}$ and $1_{2}^{b}$.

A relationship between inflection points and double tangents for simple closed curves in the real projective plane with a suitable convexity is given in [10. Finally, we formulate several open questions on the invariants $I(\gamma)$ and $\delta(\gamma)$ :

Question 1. Does $\mu(\gamma)=0$ imply $I(\gamma)=0$ ?

Question 2. Is there a generic closed curve satisfying $I(\gamma)>\mu(\gamma)$ ?

The authors do not know of any such examples. If we suppose $I(\gamma)=\mu(\gamma)$, then (2.3) yields the inequality $I(\gamma) \leq 2 \#_{\gamma}$.

Question 3. Does $I(\gamma) \leq 2 \#_{\gamma}$ hold for any generic closed curve in $\boldsymbol{R}^{2}$ ?

Question 4. Is an arbitrary locally convex curve $g$-tight?

Question 5. Find a criterion for $g$-tightness. For example, can one determine $\delta(\gamma)$ when $\gamma$ is of type $2_{2}$ or $1_{2}^{b}$ ?

\section{TABles of CURVES}
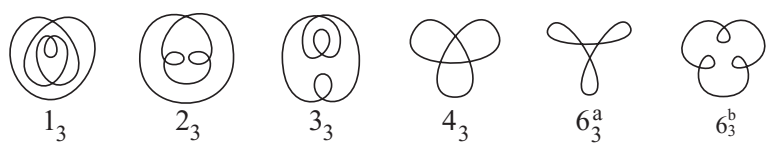

Figure 13. Curves with $I_{\gamma}=0$ and $\#_{\gamma}=3$ (6 curves in total). 


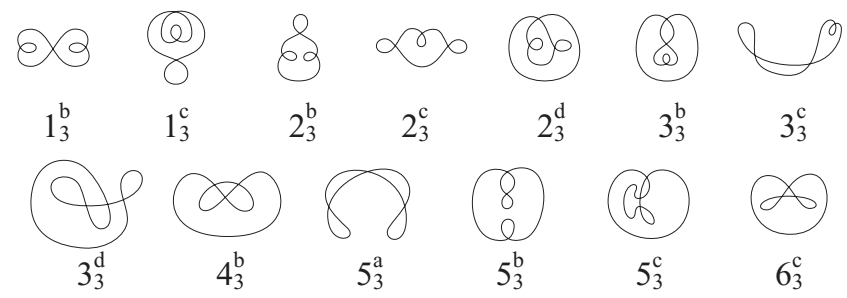

Figure 14. Curves with $I(\gamma)>0$ and $\#_{\gamma}=3$ (13 curves in total).

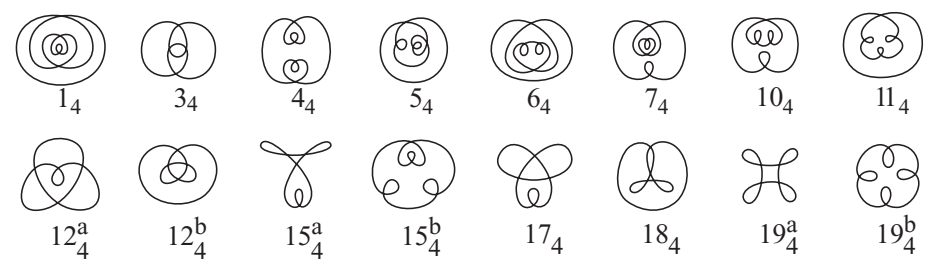

Figure 15. Curves with $I(\gamma)=0$ and $\#_{\gamma}=4$ (16 curves in total).

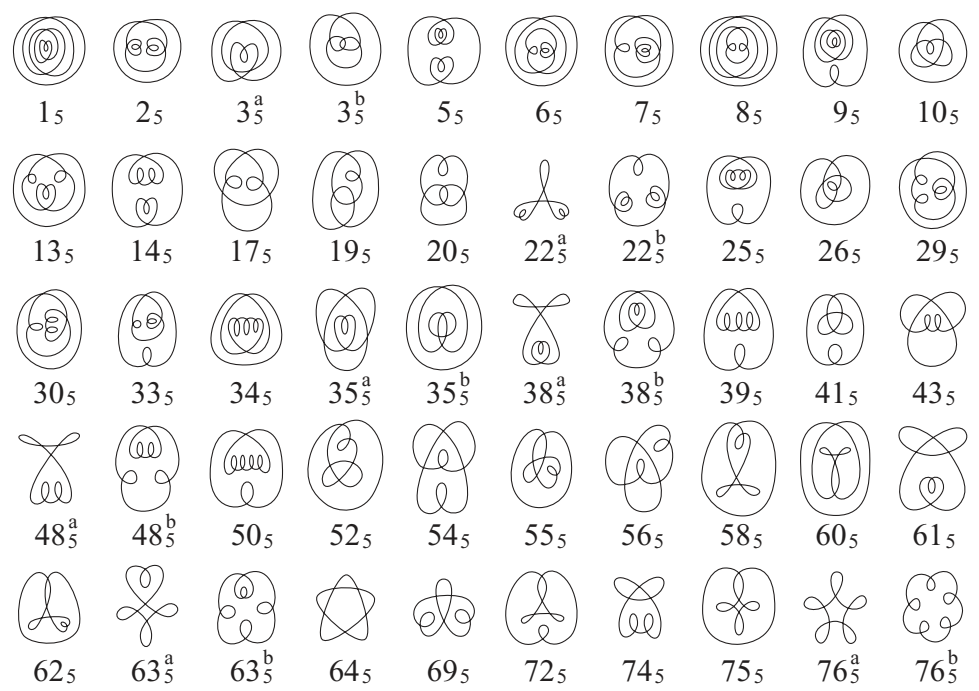

Figure 16. Curves with $I(\gamma)=0$ and $\#_{\gamma}=5$ (50 curves in total). 


\section{ACKNOWLEDGEMENTS}

The authors thank the referee and Wayne Rossman for a careful reading of the first draft and for giving valuable comments.

\section{REFERENCES}

[1] V. I. Arnold, Topological Invariants of Plane Curves and Caustics, University Lecture Series 5, American Mathematical Society, Providence, Rhode Island, 1994. MR.1286249 (95h:57003)

[2] G. Cairns and D. M. Elton, The planarity problem for signed Gauss words, J. Knot Theory Ramifications 2 (1993), 359-367. MR1247573 (95a:57032)

[3] Fr. Fabricius-Bjerre, On the double tangents of plane closed curves, Math. Scand. 11 (1962), 113-116. MR0161231(28:4439)

[4] J. S. Carter, Classifying immersed curves, Proc. Amer. Math. Soc. 111 (1991), 281-287. MR.1043406 (91d:57002)

[5] B. Halpern, Global theorems for closed plane curves, Bull. Amer. Math. Soc. 76 (1970), 96-100. MR0262936 (41:7541)

[6] B. Halpern, An inequality for double tangents, Proc. Amer. Math. Soc. 76 (1979), 133-139. MR534404(80h:53002)

[7] O. Kobayashi and M. Umehara, Geometry of scrolls, Osaka J. Math. 33 (1996), 441-473. MR:1416058 (97k:53002)

[8] T. Ozawa, On Halpern's conjecture for closed plane curves, Proc. Amer. Math. Soc. 92 (1984), 554-560. MR760945 (86a:53001)

[9] T. Ozawa, Topology of Planar Figures, Lecture Notes in Math. 9, Bifukan Inc. (in Japanese) 92 (1997).

[10] G. Thorbergsson and M. Umehara, Inflection points and double tangents on anti-convex curves in the real projective plane, Tohoku Math. J. 60 (2008), 149-181. MR.2428859 (2009c:53110)

[11] M. Umehara, 6-vertex theorem for closed planar curve which bounds an immersed surface with non-zero genus, Nagoya Math. J. 134 (1994), 75-89. MR1280654 (95e:53007)

[12] H. Whitney, On regular closed curves in the plane, Compositio Math. 4 (1937), 276-284. MR 1556973

Department of Mathematics, Kyoto Koka Senior High School, Nishi-kyogoku NodaCHO, КҮOTO 615-0861, JAPAN

E-mail address: tkmskr0329@yahoo.co.jp

Department of Mathematics, Meijo University, Tempaku, Nagoya, 468-8502 Japan

E-mail address: ozawa@meijo-u.ac.jp

Department of Mathematical and Computing Sciences, Tokyo Institute of TechNOlOGy, 2-12-1-W8-34, O-OKayama Meguro-KU, TOKYo 152-8552, JAPAN

E-mail address: umehara@is.titech.ac.jp 